\title{
Approximation of most penetrating particle size for fibrous filters considering Cunningham slip correction factor
}

\author{
Chang Hoon Jung ${ }^{\dagger}$, Young Jun Yoon ${ }^{2}$, Junshik $\mathrm{Um}^{3}$, Seoung Soo Lee ${ }^{4}$, Ji Yi Lee ${ }^{5}$, Sen Chiao ${ }^{6}$, \\ Yong Pyo $\mathrm{Kim}^{7}$
}

${ }^{1}$ Department of Health Management, Kyungin Women's University, Incheon 21041, Republic of Korea

${ }^{2}$ Korea Polar Research Institute, 26, Songdomirae-ro, Yeonsu-gu, Incheon 21990, Republic of Korea

${ }^{3}$ Department of Atmospheric Sciences, Pusan National University, Busan 46241, Republic of Korea

${ }^{4}$ Earth System Science Interdisciplinary Center, University of Maryland, College Park, MD 20740, USA

${ }^{5}$ Department of Environmental Science and Engineering, Ewha Womans University, Seoul 03760, Republic of Korea

${ }^{6}$ Center for Applied Atmospheric Research and Education, San Jose State University, San Jose, CA 95192, USA

'Department of Chemical Engineering and Material Science, Ewha Womans University, Seoul 03760, Republic of Korea

\section{ABSTRACT}

In the estimation of the aerosol single fiber efficiency using fibrous filters, there is a size range, where the particles penetrate most effectively through the fibrous collectors, and corresponding minimum single fiber efficiency. For small particles in which the diffusion mechanism is dominant, the Cunningham slip correction factor $\left(\mathrm{C}_{c}\right)$ affects the single fiber efficiency and the most penetrating particle size (MPPS). Therefore, for accurate estimation, $\mathrm{C}_{\mathrm{c}}$ is essential to be considered. However, many previous studies have neglected this factor because of its complexity and the associated difficulty in deriving the appropriate parameterization particularly for the MPPS. In this study, the expression for the MPPS, and the corresponding expression for the minimum single fiber efficiency are analytically derived, and the effects of $\mathrm{C}_{\mathrm{c}}$ are determined. In order to accommodate the slip factor for all particle-size ranges, $C_{c}$ is simplified and modified. Overall, the obtained analytical expression for the MPPS is in a good agreement with the exact solution.

Keywords: Aerosol single fiber efficiency, Cunningham slip correction factor, Fibrous collector, Minimum single fiber efficiency, Most penetrating particle size

\section{Introduction}

The collection efficiency of aerosol particles by fibrous collectors has been investigated by numerous theoretical, numerical, and experimental studies. The filtration mechanisms of fibrous collectors include Brownian diffusion, interception, inertial impaction, and gravitational settling [1-3].

Diffusion and interception (gravitational settling) dominate particle motion at the opposite size regimes. For example, an increase in particle size increases the single fiber efficiency owing to interception and gravitational settling [2-4], whereas a decrease in particle size enhances the single fiber efficiency related to Brownian diffusion. Between diffusion-dominant regime and interception-dominant regime, there exists a size range where none of the diffusion or interception (or gravitational settling) is predominant but they operate simultaneously. The size regime exists where aerosol particles penetrate most effectively through the fibrous collectors [5-7].

For small particle sizes where Brownian diffusion is dominant, Cunningham slip correction factor $\left(\mathrm{C}_{\mathrm{c}}\right)$ and Knudsen number $(\mathrm{Kn})$ play an important role in estimating accurate filtration efficiency. In cases where the aerosol-particle size in air approaches the mean free path of gas molecules, the discontinuities of the medium should be considered. $\mathrm{C}_{\mathrm{c}}$ accounts for these discontinuities and can be useful for describing aerosol properties, such as sedimentation and diffusion [1, 8-10]. For the interaction of gases and particles, Kn, the ratio of mean free path $(\lambda)$ to particle diameter $\left(d_{p}\right)$, is widely used [11].
This is an Open Access article distributed under the terms of the Creative Commons Attribution Non-Commercial License (http://creativecommons.org/licenses/by-nc/3.0/) which permits unrestricted non-commercial use, distribution, and reproduction in any medium, provided the original work is properly cited.

Copyright (C) 2020 Korean Society of Environmental Engineers
Received February 13, 2019 Accepted April 12, 2019

${ }^{\dagger}$ Corresponding author

Email: jch@kiwu.ac.kr

Tel: +82-32-540-0166 Fax: +82-32-540-0167

ORCID: 0000-0003-0221-6764 
Among many filtration studies, Jung and Lee [7] obtained an approximated analytical solution for the most penetrating particle size (MPPS) in a multiple fluid sphere system. In their study, a harmonic-mean type approximation was applied, and the obtained solution was compared with the numerically calculated results. However, $C_{c}$ was not considered because the derivation of an analytical expression considering this factor was complicated.

Lee and Liu [2] derived equations for predicting the minimum efficiency and the MPPS for fibrous filters, considering the diffusion and interception mechanisms. Their results showed that the MPPS decreased with increases in filtration velocity and fiber volume fraction, and increased with an increase in filter fiber size. In addition, they obtained a corresponding equation for the minimum efficiency, and demonstrated the dependence of the MPPS on the filtration velocity, fiber volume fraction, and fiber size. They approximated $C_{c}$ for different ranges of $\mathrm{Kn}$, with three different formulas, which exhibited a good correlation with an original equation within specified ranges. However, these approximated equations are limited because they are valid only for a particular size range. However, in the diffusion-mechanism-dominant regime for small particle sizes, aerosol slip effects are non-negligible, and should be corrected using $C_{c}$.

In this study, a new expression for the MPPS is analytically derived, and $C_{c}$ is simplified and modified to accommodate all the particle-size ranges. The newly derived expression includes the slip correction, which is implicitly considered in the diffusion coefficient for aerosol particles with low Kn values.

\section{Theoretical Backgrounds}

Generally, $C_{c}$, can be expressed as follows [8]:

$$
C_{c}=1+\frac{\lambda}{d_{p}}\left(2.514+0.8 \exp \left(-0.55 \frac{d_{p}}{\lambda}\right)\right)
$$

Where $\lambda$ is the mean free path of air molecules as a constant, and $d_{p}$ is the particle diameter. However, this expression is complex, and requires simplification for parameterization studies [1, 12]. One of the simplified expressions for $C_{c}$, used in many studies, is as follows [2]:

$$
C_{c}=1+3.34 \frac{\lambda}{d_{p}}
$$

The simplified expression for $C_{c}$ (Eq. (2)) is simple and sufficiently accurate compared to original expression (Eq. (1)) [2]. For example, the diffusion coefficient $(D)$ and Peclet number $(P e)$, considering $C_{c}$, can be expressed as follows:

$$
\begin{gathered}
D=\frac{k T C_{c}}{3 \pi \mu d_{p}} \cong \frac{k T}{3 \pi \mu d_{p}}\left(1+3.34 \frac{\lambda}{d_{p}}\right) \\
P e^{-2 / 3}=\left(\frac{2 a u}{D}\right)^{-2 / 3}=\left(\frac{D}{D_{f} u}\right)^{2 / 3} \\
=\left(\frac{1}{D_{f} u}\right)^{2 / 3}\left(\frac{k T}{3 \pi \mu d_{p}}\right)^{2 / 3}\left(1+3.34 \frac{\lambda}{d_{p}}\right)^{2 / 3}
\end{gathered}
$$

where $k$ is the Boltzmann constant, $T$ is the absolute temperature, is the viscosity of air, $D_{f}$ is the diameter of the filter, and $u$ is the flow velocity. Subsequently, the aerosol single fiber efficiency $\left(\eta_{D}\right)$ in association with the diffusion mechanism can be expressed as follows:

$$
\begin{aligned}
\eta_{D} & =2.6\left(\frac{1-\alpha}{K}\right)^{1 / 3} P e^{-2 / 3}=2.6\left(\frac{1-\alpha}{K}\right)^{1 / 3}\left(\frac{k T C_{c}}{3 \pi \mu d_{p} u D_{f}}\right)^{2 / 3} \\
& =2.6\left(\frac{1-\alpha}{K}\right)^{1 / 3}\left(\frac{k T}{3 \pi \mu d_{p} u D_{f}}\right)^{2 / 3}\left(1+3.34 \frac{\lambda}{d_{p}}\right)^{2 / 3}
\end{aligned}
$$

Here, $K$ is the hydrodynamic factor of the Kuwabara flow $\left(=-\frac{1}{2} \ln \alpha-\frac{3}{4}+\alpha-\frac{\alpha^{2}}{4}\right)$, and $\alpha$ is the volume fraction, solidity, or packing density of the filter [13].

Eq. (5) can be further simplified via the approximation of $\left(1+3.34 \frac{\lambda}{d_{p}}\right)^{2 / 3}$. Hence, Lee and Liu [2] approximated $C_{c}$ for three different size ranges, as follows:

$C_{c}=1$, for large particles with a continuum-flow-regime

$$
\text { assumption }\left(\frac{\lambda}{d_{p}} \ll 1\right) \text {; }
$$

$C_{c}=3.33 \frac{\lambda}{d_{p}}$, for very small particles $\left(\frac{\lambda}{d_{p}} \gg 1\right)$;

$C_{c}=3.69\left(\frac{\lambda}{d_{p}}\right)^{1 / 2}$, for particles with intermediate sizes.

Although these approximations demonstrate good correlations with the original equation, the approximated equations are valid only for the given size conditions and require different approximations in the other size ranges. For example, the single fiber efficiency can be generally expressed using a combination of the single fiber efficiency of diffusion and interception. If these three types of $C_{c}$ are considered, the filtration single fiber efficiency should be expressed by three different formulas. Moreover, if the minimum single fiber efficiency as well as the corresponding $d_{p}$ varies for different formulas, the estimation of the filtration-mechanism characteristics considering $C_{c}$ becomes confusing; therefore, this factor was neglected in several previous studies. Thus, for practical purposes, a more general approximation that covers all size ranges is required, considering the continuous changes in $C_{c}$.

\section{Results and Discussion}

\subsection{New Approximated Expression for the Cunningham Slip Correction Factor and Single Fiber Efficiency}

According to Lee and Liu [14] and Eq. (5), the single fiber effi- 
ciency $\left(\eta_{D}\right)$ due to diffusion can be expressed in proportion to $C_{c}^{2 / 3}\left(\eta_{D} \propto C_{c}^{2 / 3}\right)$. Thus, for the further evolution of the analytical expression for the MPPS, $C_{c}$ should be simplified. In this study, we evaluated $C_{c}^{2 / 3}$ using the $1^{\text {st }}$ order Taylor series expansion as a function of the $d_{p}$ :

$$
C_{c}^{2 / 3} \cong\left(1+3.34 \frac{\lambda}{d_{p}}\right)^{\frac{2}{3}} \cong \zeta\left\{1+\frac{2}{3}\left(3.34 \frac{\lambda}{d_{p}}\right)\right\}
$$

Then,

$$
C_{c} \cong \zeta^{3 / 2}\left\{1+\frac{2}{3}\left(3.34 \frac{\lambda}{d_{p}}\right)\right\}=\vartheta\left\{1+\frac{2}{3}\left(3.34 \frac{\lambda}{d_{p}}\right)\right\}^{\frac{3}{2}}
$$

Here, we adapted the correlation factors, and , based on the comparison results between the real and approximated formulas:

$$
\vartheta=\zeta^{3 / 2}=0.6\left(\frac{d_{p}}{\lambda}\right)^{0.1}
$$

Subsequently, $D$ and pe can be obtained using Eq. (3), (5), (8), and (9), as follows:

$$
\begin{gathered}
D=\frac{k T C_{c}}{3 \pi \mu d_{p}} \cong \frac{\vartheta k T}{3 \pi \mu d_{p}}\left\{1+\frac{2}{3}\left(3.34 \frac{\lambda}{d_{p}}\right)\right\}^{\frac{3}{2}} \\
=0.6\left(\frac{d_{p}}{\lambda}\right)^{0.1} \frac{k T}{3 \pi \mu d_{p}}\left\{1+\frac{2}{3}\left(3.34 \frac{\lambda}{d_{p}}\right)\right\}^{\frac{3}{2}} \\
D^{2 / 3}=\left(\frac{k T C_{c}}{3 \pi \mu d_{p}}\right)^{2 / 3} \cong\left\{0.6\left(\frac{d_{p}}{\lambda}\right)^{0.1}\right\}^{2 / 3}\left(\frac{k T}{3 \pi \mu d_{p}}\right)^{2 / 3}\left\{1+\frac{2}{3}\left(3.34 \frac{\lambda}{d_{p}}\right)\right\} \\
P e^{-2 / 3}=\left(\frac{D_{f} u}{D}\right)^{-2 / 3}=\left(\frac{D}{D_{f} u}\right)^{2 / 3}=\left\{0.6\left(\frac{d_{p}}{\lambda}\right)^{0.1}\right\}^{2 / 3}\left\{1+\frac{2}{3}\left(3.34 \frac{\lambda}{d_{p}}\right)\right\} \\
=(0.6)^{2 / 3}\left(\frac{d_{p}}{\lambda}\right)^{1 / 15}\left(\frac{k T}{3 \pi \mu d_{p} D_{f} u}\right)^{2 / 3}\left\{1+\frac{2}{3}\left(3.34 \frac{\lambda}{d_{p}}\right)\right\}
\end{gathered}
$$

Further, the $\eta_{D}$ for diffusion can be expressed as a linear function of the $d_{p}$, as in Eq. (5) and (10).

Table 1 depicts the expressions for the single fiber efficiency by diffusion; the previous expression by Lee and Liu [2], and the currently derived expression are presented. As seen in Table 1, the currently derived expression can be used for the entire size range. However, different formulas have to be used for different size ranges when applying Lee and Liu's expression.

Fig. 1 shows the comparison of the exact values and the newly developed approximated solutions in this study, for $C_{c}, D$, and $P e$, respectively; the results are in good agreement with each other. Here, the "exact" $C_{c}$ refers to values calculated from Eq. (1).

Table 1. Comparison of the Expressions for the Single Fiber Efficiency by Diffusion (Previous Expression vs. Currently Derived Expression)

\section{Previous expression [2]}

\section{Current expression}

$$
\text { - } 2.6\left(\frac{1-\alpha}{K}\right)^{1 / 3}\left(\frac{k T}{3 \pi \mu d_{p} u D_{f}}\right)^{2 / 3} \text { for } \frac{\lambda}{d_{p}} \ll 1
$$

Single fiber
efficiency by $\cdot 2.6\left(\frac{1-\alpha}{K}\right)^{1 / 3}\left(\frac{3.33 k T \lambda}{3 \pi \mu d_{p}^{2} u D_{f}}\right)^{2 / 3}$ for $\frac{\lambda}{d_{p}} \gg 1$

For the entire size range,

$$
\text { - } 2.6\left(\frac{1-\alpha}{K}\right)^{1 / 3}\left(\frac{k T C_{c}}{3 \pi \mu d_{p}^{2} u D_{f}}(\sqrt{3.69 \lambda})\right)^{2 / 3}
$$$$
\begin{aligned}
& 2.6(0.6)^{2 / 3}\left(\frac{1-\alpha}{K}\right)^{1 / 3}\left(\frac{1}{\lambda}\right)^{1 / 15}\left(\frac{k T}{3 D_{f} u \pi \mu}\right)^{2 / 3} d_{p}^{-3 / 5} \\
& +\frac{5.2(3.34)(0.6)^{2 / 3}}{3}\left(\frac{1-\alpha}{K}\right)^{1 / 3}(\lambda)^{14 / 15}\left(\frac{k T}{3 D_{f} u \pi \mu}\right)^{2 / 3} d_{p}^{-8 / 5}
\end{aligned}
$$

for intermediate size.
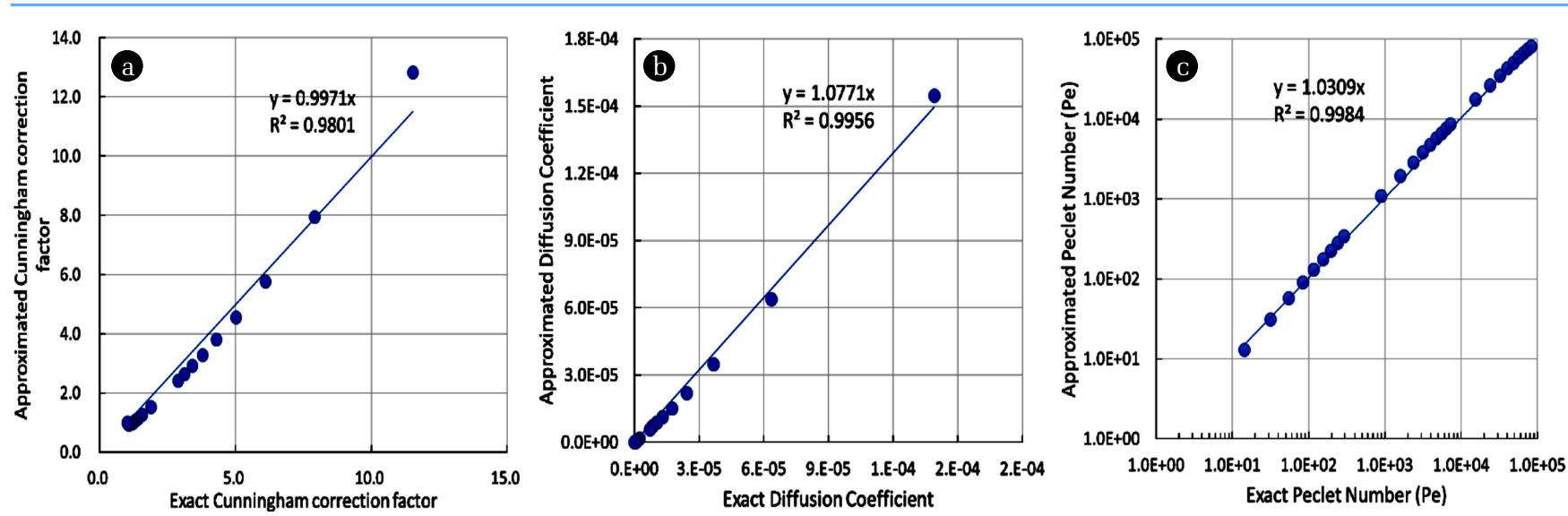

Fig. 1. Comparison of the exact and approximated (a) Cunningham slip correction factors, (b) Diffusion coefficients, and (c) Peclet numbers. 

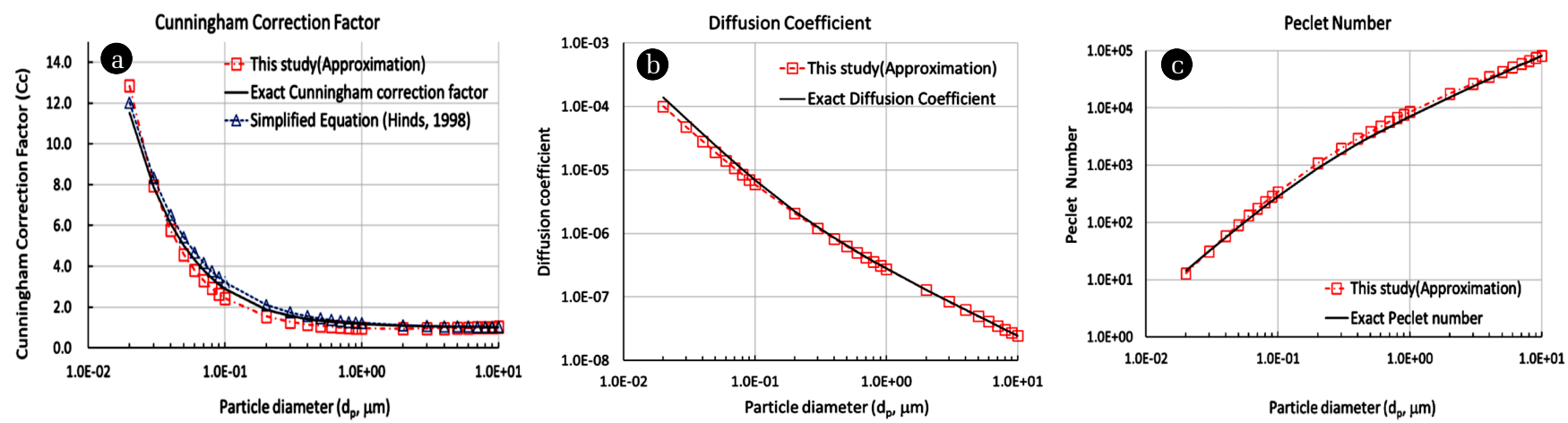

Fig. 2. Comparison of the exact and approximated (a) Cunningham slip correction factors, (b) Diffusion coefficients, and (c) Peclet numbers, as functions of the particle diameter $\left(D_{f}=0.02 \mathrm{~mm}\right)$.

The correlation coefficients are 0.9801, 0.9956, and 0.9984 with slopes of $0.9971,1.0771$, and 1.0309 for $C_{c}, D$, and $P$, respectively.

Further, the total $\eta_{D}$, including that owing to interception and gravitational settling, is expressed as a function of the $d_{p}$ :

$$
\begin{aligned}
\eta_{T}= & \eta_{D}+\eta_{R}+\eta_{G} \\
= & 2.6(0.6)^{2 / 3}\left(\frac{1-\alpha}{K}\right)^{1 / 3}\left(\frac{1}{\lambda}\right)^{1 / 15}\left(\frac{k T}{3 D_{f} u \pi \mu}\right)^{2 / 3} d_{p}^{-3 / 5} \\
& +\frac{5.2(3.34)(0.6)^{2 / 3}}{3}\left(\frac{1-\alpha}{K}\right)^{1 / 3}(\lambda)^{14 / 15} \\
& \left(\frac{k T}{3 D_{f} u \pi \mu}\right)^{2 / 3} d_{p}^{-8 / 5}+\left(\frac{1-\alpha}{K}\right)\left(\frac{d_{p}}{D_{f}}\right)^{2}+\frac{\rho_{p} d_{p}^{2} g}{18 \mu u}
\end{aligned}
$$

where $\eta_{R}$ and $\eta_{G}$ are the single fiber efficiencies due to interception and gravitational settling, respectively.

$$
\begin{aligned}
& \eta_{R}=\left(\frac{1-\alpha}{K}\right) \frac{R^{2}}{1+R} \cong\left(\frac{1-\alpha}{K}\right) R^{2}=\left(\frac{1-\alpha}{K}\right)\left(\frac{d_{p}}{D_{f}}\right)^{2}, \\
& \eta_{G}=\frac{\rho_{p} d_{p}^{2} g}{18 \mu u}
\end{aligned}
$$

where $R$ is the interception parameter $\left(=\frac{d_{p}}{D_{f}}\right)$ and $g$ is the gravitational coefficient. It should be noted that the newly approximated diffusion single fiber efficiency $\left(\eta_{D}\right)$ in Eq. (11) can now be expressed as a linear combination of the $d_{p}$.

Based on Fig. 1, we conclude that the approximated analytical expression for $C_{C}$ estimates the exact $C_{c}$, and related parameters, such as $D$ and $P e$, with good confidence. Fig. 2 shows $C_{c}, D$, and $P e$ as functions of the $d_{p}$. The exact values of $C_{c}$ which are calculated using Eq. (1) is compared with the approximated values calculated using Eq. (10). The filter diameter $\left(D_{f}\right)$ is set at $0.02 \mathrm{~mm}$, and the packing density and flow velocity are set at 0.08 and $1 \mathrm{~cm} / \mathrm{s}$, respectively. The results exhibit a good agreement (Fig. 2).

Fig. 3 shows the total single fiber efficiency as a function of the $d_{p}$ for different assumptions of $C_{c}$. For a filter diameter of 0.02 $\mathrm{mm}\left(D_{f}=0.02 \mathrm{~mm}\right)$, and flow velocities of $1 \mathrm{~cm} / \mathrm{s}$ and $10 \mathrm{~cm} / \mathrm{s}$, the values of the single fiber efficiencies for the exact $C_{c}, C_{c}=1$, and the approximated $C_{C}$ from this study are compared. As seen
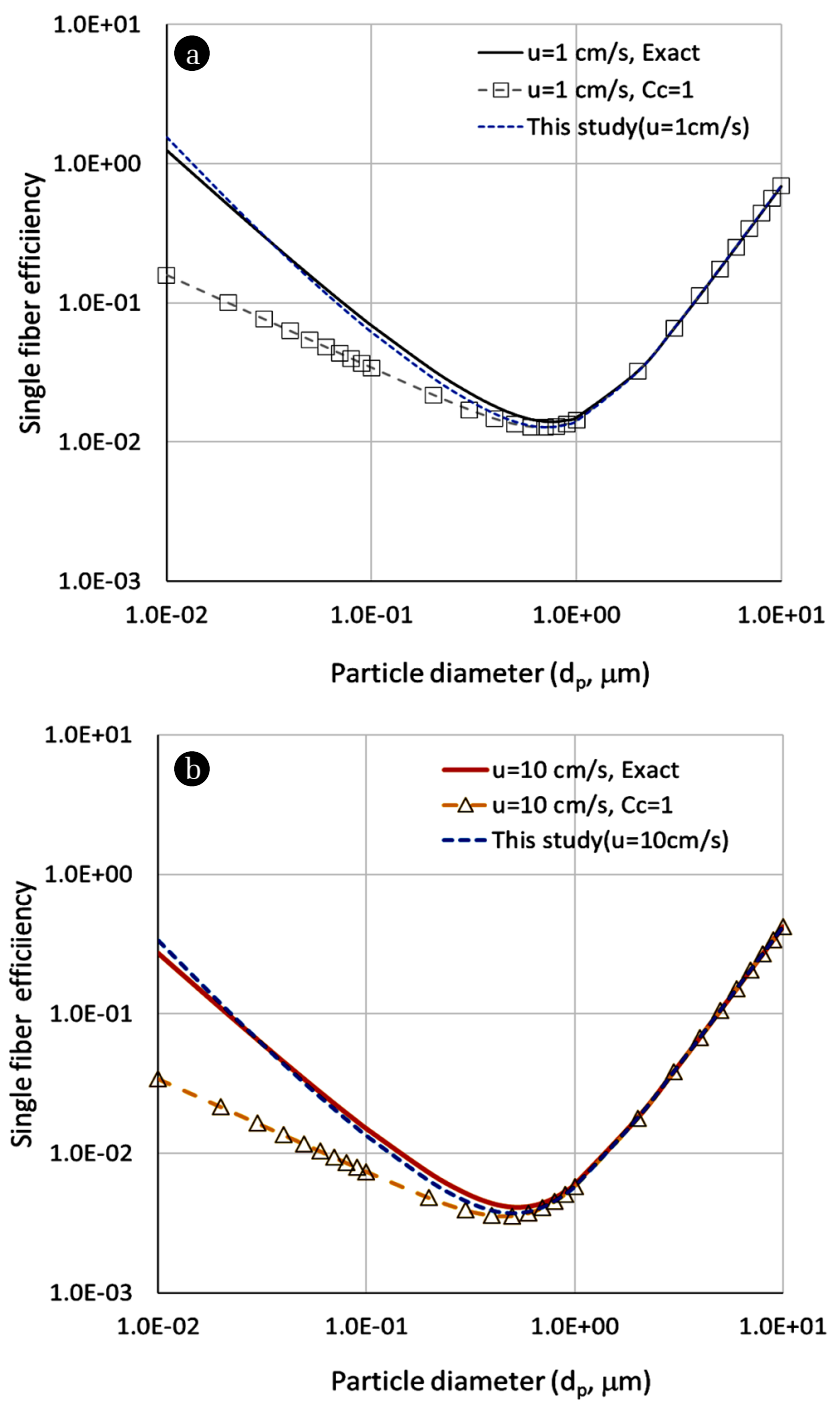

Fig. 3. Single fiber efficiency as a function of the particle diameter for different Cunningham slip correction factors $\left(C_{c}=1\right.$, exact solution with Eq. (1), and approximated value in this study; $\left.D_{f}=0.02 \mathrm{~mm}\right)$. 
in Fig. 3, the approximated solutions of the single fiber efficiencies using the approximated $C_{c}$ agree with those using the exact $C_{c}$. The single fiber efficiency with unity $C_{c}$ exhibits a discrepancy compared to the exact values, increasing with the decreasing particle size.

\subsection{Analytic Expression for the MPPS of Fibrous Filters}

As explained in the introduction, an increase in the particle size increases the single fiber efficiency due to interception and gravitational settling, whereas a reduction in the particle size causes an increase in the single fiber efficiency because of Brownian diffusion. It is notable that there is a regime with an intermediate particle size, where two or more mechanisms operate simultaneously, but no mechanism predominates over others. In this regime, the single fiber efficiency is minimum. The minimum single fiber efficiency diameter or the MPPS can be acquired by the differentiation of the single fiber efficiency with respect to the particle size, and the acquired value can be used to establish a value of zero. Previous studies determined the MPPS and the corresponding minimum single fiber efficiency with no consideration of $C_{C}$ or with complex expressions [15-16]. Thus, the effects of $C_{c}$ and the corresponding expression for the minimum single fiber efficiency, as well as the MPPS, must be improved in a way that considers $C_{c}$ and simplifies those expressions. In this study, we approximated $C_{c}$ to accurately estimate the single fiber efficiency and MPPS, as well as the minimum single fiber efficiency.

The consequent equation for the MPPS of fibrous filters can be obtained as follows, by differentiating Eq. (11) with respect to the $d_{p}$ :

$$
\begin{aligned}
& \frac{\partial \eta_{T}}{\partial d_{p}}=\frac{-3(2.6)(0.6)^{2 / 3}}{3 \pi \mu d_{p}}\left(\frac{1-\alpha}{K}\right)^{1 / 3} \lambda^{-1 / 15}\left(\frac{k T}{3 D_{f} u \pi \mu}\right)^{2 / 3} d_{p}^{-\frac{8}{5}} \\
& -\frac{8(5.2)(3.34)(0.6)^{2 / 3}}{(3)(5)}\left(\frac{1-\alpha}{K}\right)^{1 / 3} \lambda^{14 / 15}\left(\frac{k T}{3 D_{f} u \pi \mu}\right)^{2 / 3} d_{p}^{-13 / 5} \\
& \quad+\left\{2\left(\frac{1-\alpha}{K}\right)\left(\frac{1}{D_{f}}\right)^{2}+\frac{\rho_{p} g}{9 \mu u}\right\} d_{p}=0
\end{aligned}
$$

Eq. (13) can be expressed in a simpler form as follows:

$$
\frac{\partial \eta_{T}}{\partial d_{p}}=\varphi_{1} d_{p}^{-8 / 5}+\varphi_{2} d_{p}^{-13 / 5}+\varphi_{3} d_{p}=0 .
$$

where

$$
\begin{aligned}
\varphi_{1} & =-\frac{3(2.6)(0.6)^{2 / 3}}{3 \pi \mu d_{p}}\left(\frac{1-\alpha}{K}\right)^{1 / 3} \lambda^{-1 / 15}\left(\frac{k T}{3 D_{f} u \pi \mu}\right)^{2 / 3} \\
\varphi_{2} & =-\frac{8(5.2)(3.34)(0.6)^{2 / 3}}{(3)(5)}\left(\frac{1-\alpha}{K}\right)^{1 / 3} \lambda^{14 / 15}\left(\frac{k T}{3 D_{f} u \pi \mu}\right)^{2 / 3} \\
\varphi_{3} & =\left\{2\left(\frac{1-\alpha}{K}\right)\left(\frac{1}{D_{f}}\right)^{2}+\frac{\rho_{p} g}{9 \mu u}\right\}
\end{aligned}
$$

The first and second terms $\left(\varphi_{1}\right.$ and $\left.\varphi_{2}\right)$ refer to the diffusion, and the third term $\left(\varphi_{3}\right)$ refers to the interception, and gravitational settling mechanisms in Eq. (14). The single fiber efficiency due to diffusion increases as the $d_{p}$ decreases, while that due to interception increases as the $d_{p}$ increases. Thus, the leading terms can be estimated for each dominant particle or collector-size region. If the two dominant terms in Eq. (14) are determined, the resulting approximated equations can be generated as follows $[6,7,16]$ :

$$
\begin{aligned}
& \varphi_{1} d_{p}^{8 / 5}+\varphi_{3} d_{p}=0 \\
& \varphi_{2} d_{p}^{13 / 5}+\varphi_{3} d_{p}=0 .
\end{aligned}
$$

The subsequent solutions from Eq. (15) are expressed as follows:

$$
d_{s 1}=\left(\frac{-\varphi_{1}}{\varphi_{3}}\right)^{13 / 5}, d_{s 2}=\left(\frac{-\varphi_{2}}{\varphi_{3}}\right)^{5 / 18}
$$

Further, the minimum scavenging coefficient particle size is expressed as in Eq. (17) by applying the harmonic-mean type approximation [16]:

$$
d_{p, \min }=\bar{\omega} \sqrt{\frac{1}{\frac{1}{d_{s 1}^{2}}+\frac{1}{d_{s 2}^{2}}}}
$$

Here, the correction parameter, $\bar{\omega}$, can be adapted to minimize the errors between the exact and approximated MPPS. In this study, an $\bar{\omega}$ of 0.6 is suggested by comparing the exact and harmonic mean results.

Fig. 4 shows the MPPS as a function of the velocity, considering $D_{f}$ of $0.02 \mathrm{~mm}$ and $0.1 \mathrm{~mm}$. The MPPS can be determined in accordance with a dominant mechanism, for any given condition. As seen in Fig. 4, the MPPS increases as the filtration velocity increases up to a point, and after the point, decreases with the increasing velocity. According to the previous study [5], the MPPS shifts to a larger size as the flow velocity increases for fiber diameter $\left(d_{f}\right)$ of $0.02 \mathrm{~mm}$, when Brownian diffusion and the gravitational force are dominant. The MPPS shifts to a smaller size as the flow velocity increases for $d_{f}$ of $0.1 \mathrm{~mm}$, when Brownian diffusion and interception are dominant. As shown in Fig. 4, the MPPS obtained using the analytical expression of this study agrees well with the exact MPPS. Fig. 5 depicts the MPPS as a function of the filter packing density; $d_{f}$ of 0.02 and $0.2 \mathrm{~mm}$ are compared for filtration velocities of 1 and $10 \mathrm{~cm} / \mathrm{s}$, respectively. The actual MPPS and the one determined in this study show a good agreement. For a $D_{f}$ of $0.2 \mathrm{~mm}$, the MPPS increases with the increase in packing density up to a value

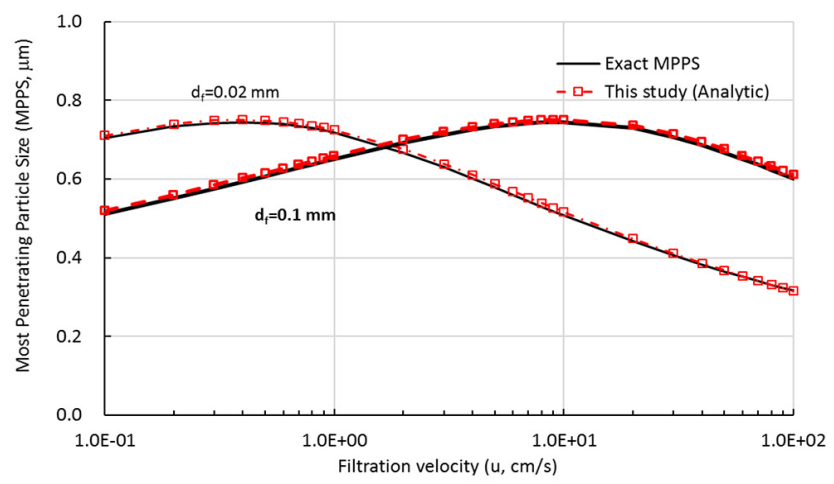

Fig. 4. Most penetrating particle size as a function of the filtration velocity for different filter diameters of $0.02 \mathrm{~mm}$ and $0.1 \mathrm{~mm}$. 


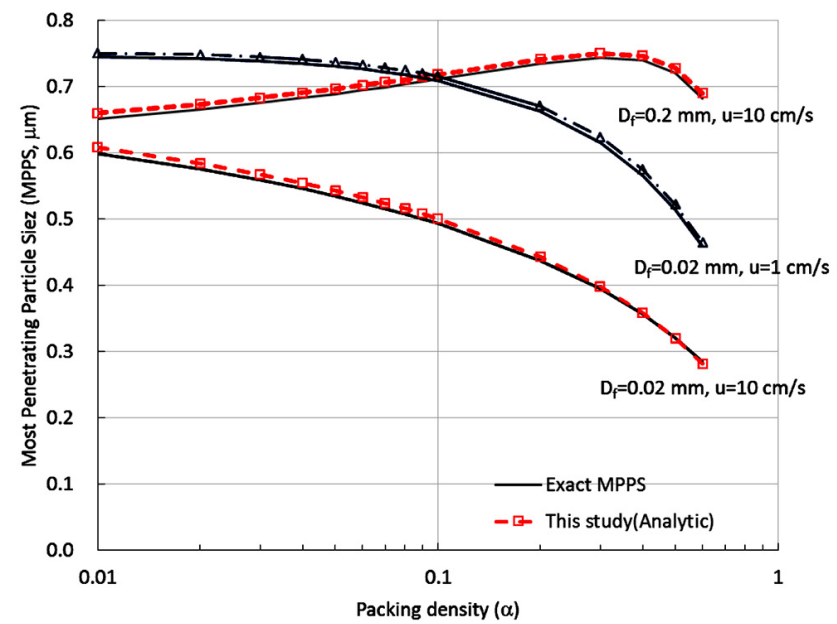

Fig. 5. Comparison of the exact solution of the most penetrating particle size with its analytical counterpart, as a function of the packing density.

of approximately 0.3, after which the MPPS starts to decrease. However, for a $D_{f}$ of $0.02 \mathrm{~mm}$, the MPPS decreases with the increase in packing density over the density range in Fig. 5.

\subsection{Minimum Single Fiber Efficiency}

Based on the MPPS, the corresponding minimum single fiber efficiency $\left(\eta_{\min }\right)$ is obtained by substituting $d_{p}$ in Eq. (11) with $d_{p, \min }$ in Eq. (17). Fig. 6 shows the minimum single fiber efficiency as a function of the filtration velocity; the exact solution and approximated one from this study are compared. The minimum single fiber efficiency decreases with the increase in filtration velocity. The minimum single fiber efficiency is greater at smaller $d_{f}$ over

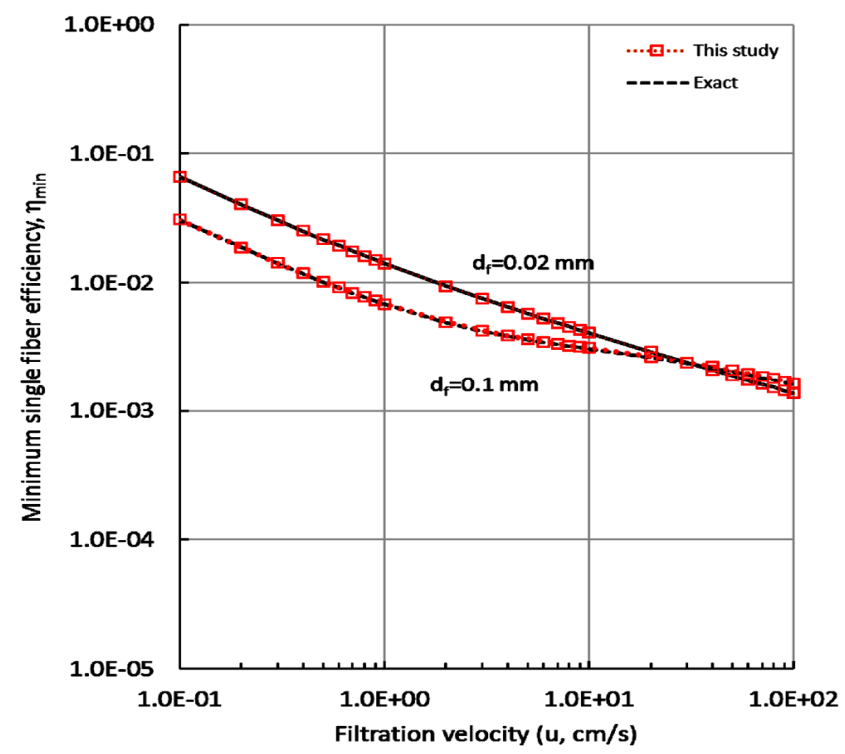

Fig. 6. Comparison of the exact solution of the minimum single fiber efficiency, as a function of the filtration velocity, with its analytical counterpart, for different filter diameters of $0.02 \mathrm{~mm}$ and $0.1 \mathrm{~mm}$. the filtration velocities up to $\sim 20 \mathrm{~cm} / \mathrm{s}$ and for lower filtration velocities. Nevertheless, the difference in the minimum single fiber efficiency between different $d_{f}$ decreases with the increase in filtration velocity.

\section{Conclusions}

The $\mathrm{C}_{c}$ is significant, for particle sizes below 2-3 $\mu \mathrm{m}$, in air at ambient conditions. In filtration studies, the effects of $C_{c}$ are critical and can be considered for the exact estimation of the collection efficiency and MPPS. For instance, in the regime where the diffusion mechanism is dominant, the aerosol slip effects cannot be neglected for small particle sizes, and the effects should be calibrated using $C_{c}$. However, the complex formula for $C_{c}$ requires a simplified expression, for parameterization studies.

In this study, an expression for the MPPS and corresponding minimum single fiber efficiency were analytically derived based on the newly developed analytically simplified $C_{C}$ expression in association with the slip effects of small particles. Slip correction was implicitly embedded in the $D$ for aerosol particles with low $\mathrm{Kn}$. The comparison of the obtained analytical solution for the MPPS, which considers the particle slip of small particles, with the exact solution demonstrated a good agreement. This result indicates that the simplified expression for $C_{c}$, demonstrated in this study, can be used for obtaining the analytical solution for the MPPS, including particles with low Knudsen numbers, with a high confidence level.

\section{Acknowledgments}

This research was supported by the Basic Science Research Program through the National Research Foundation of Korea (NRF), funded by the Ministry of Education (NRF-2018R1D1A1A09083227), and by the KOPRI program (PN19081), funded by the National Research Foundation of Korea Grant (NRF-2016M1A5A1901786).

\section{References}

1. Hinds WC. Aerosol technology, properties behavior, and measurement of airborne particles. 2nd ed. New York: John Wiley and Sons; 1998.

2. Lee KW, Liu BYH. On the minimum efficiency and the most penetrating particle size for fibrous filters. J. Air Pollut. Control Assoc. 1980;30:337-381.

3. Shou D, Fan J, Zhang H, Qian X, Ye L. Filtration efficiency of non-uniform fibrous filters. Aerosol Sci. Technol. 2015;49: 912-919.

4. Lee KW, Gieseke JA. Collection of aerosol particles by packed beds. Environ. Sci. Technol. 1979;13:466-470.

5. Lee KW. Maximum penetration of aerosol particles in granular bed filters. J. Aerosol Sci. 1981;12:79-87.

6. Jung $\mathrm{CH}$, Lee KW. Analytic solution on critical suspended particle size and minimum collection efficiency in deep bed 
filtration. J. Environ. Eng. 2006;132:1381-1386.

7. Jung $\mathrm{CH}$, Lee KW. Approximated solution for the most penetrating particle size in multiple fluid sphere systems. Environ. Eng. Sci. 2007;24:257-266.

8. Gussman RA. On the aerosol particle slip correction factor. J. Appl. Meteorol. 1969;8:999-1001.

9. Moshfegh A, Shams M, Ahmadi G, Ebrahimi R. A novel surface-slip correction for microparticles motion. Colloids Surf. A. Physicochem. Eng. Asp. 2009;345:112-120.

10. Moshfegh A, Shams M, Ahmadi G, Ebrahimi R. A new expression for spherical aerosol drag in slip flow regime. J. Aerosol Sci. 2010;41:384-400.

11. Jung CH, Lee KW. Analytical solution of unified flow field for multiple fluid collectors in finite Knudsen number regime.
Environ. Eng. Sci. 2007;24:216-227.

12. Sorensen CM, Wang GM. Note on the correction for diffusion and drag in the slip regime. Aerosol Sci. Technol. 2000;33:353-356.

13. Kuwabara S. The forces experienced by randomly distributed parallel circular cylinders or spheres in a viscous flow at small Reynolds numbers. J. Phys. Soc. Jpn. 1958;14:527-532.

14. Lee KW, Liu BYH. Theoretical study of aerosol filtration by fibrous filters. Aerosol Sci. Technol. 1982;1:147-161.

15. Lee KW. Maximum penetration of aerosol particles in granular bed filters. J. Aerosol Sci. 1981;12:79-87.

16. Jung CH, Bae SY, Kim YP. Approximated solution on the properties of the scavenging gap during precipitation using harmonic mean method. Atmos. Res. 2011;99:496-504. 